\title{
RF-Heartbeat: Robust and Contactless Heartbeat Monitoring Based on FMCW Radar
}

This paper was downloaded from TechRxiv (https://www.techrxiv.org).

LICENSE

CC BY-NC-SA 4.0

SUBMISSION DATE / POSTED DATE

20-07-2021 / 22-07-2021

CITATION

Pu, Jian; Zhang, Hao (2021): RF-Heartbeat: Robust and Contactless Heartbeat Monitoring Based on FMCW Radar. TechRxiv. Preprint. https://doi.org/10.36227/techrxiv.15021645.v1

DOI

10.36227/techrxiv.15021645.v1 


\title{
RF-Heartbeat: Robust and Contactless Heartbeat Monitoring Based on FMCW Radar
}

\author{
Pu Jian, Hao Zhang, Changyu Liu, Yicheng Yao, Peng Wang, Baiju Yan, Xianxiang Chen, Lidong Du, \\ Chengyu Zhuang and Zhen Fang
}

\begin{abstract}
Objective: Heartbeat Monitoring is significant in clinical space and daily life. Traditionally, heartbeat monitoring requires users to wear sensors, which could cause discomfort. The past decade has witnessed wireless vital signs monitoring becoming more and more feasible. As a contactless approach, it can relieve users from wearing devices and follow specific instructions. In addition, by monitoring remotely, it could protect clinician from cross-infection, especially in the pandemic. We propose RF-Heartbeat, a robust heartbeat monitoring system based on FMCW radar, enabling accurate inter-beat interval (IBI) estimation. Methods: Dynamic programming is implemented to improve the robustness of the template matching algorithm. Template matching requires a high similarity of heartbeat shape and suffers from shape variation of heartbeat signal obtained by radar. With heartbeat signal, generated template, and heart rate variability (HRV) prior knowledge, global optimal heart segmentation can be achieved to solve this problem. In addition, an optimal range bin selection algorithm is proposed. Therefore, when the user is in different positions and postures, RF-Heartbeat can always obtain high-quality heartbeat signals. Results: In the heartbeat monitoring experiment for a total of 72 hours and 56 minutes involving 10 participants, under time coverage of $91.2 \%$, the median error of IBI was $12 \mathrm{~ms}$. Conclusion: RFHeartbeat achieves a higher performance compared with stateof-the-art heartbeat monitoring systems. Besides high accuracy, RF-Heartbeat has strong robustness to different individuals in different postures and positions. It enables accurate long-term heartbeat monitoring and has wide applications in clinical space
\end{abstract}

This work was supported by the National Key Research and Development Project 2020YFC1512304, 2020YFC2003703, 2018YFC2001101, 2018YFC2001802, CAMS Innovation Fund for Medical Sciences2019-I2M5-019, and National Natural Science Foundation of China (Grant 62071451). (Corresponding authors: Lidong Du; Chengyu Zhuang; Zheng Fang.)

$\mathrm{Pu}$ Jian and Hao Zhang contributed equally to this work.

Pu Jian, Hao Zhang, Changyu Liu, Yicheng Yao are with the Aerospace Information Research Institute, Chinese Academy of Sciences, Beijing 100190, China, and also with the School of Electronic, Electrical and Communication Engineering, University of Chinese Academy of Sciences, Beijing 100049 China (jianpu19@mails.ucas.ac.cn; lcyu3@163.com; zhanghao190@mails.ucas.ac.cn; e-mail: yaoyicheng19@mails.ucas.ac.cn).

Baiju Yan is with the School of Electronic Information and Electrical Engineering, Shanghai Jiao Tong University, Shanghai 200240, China (e-mail: yanbaiju@ sjtu.edu.cn).

Peng Wang, Lidong Du and Xianxiang Chen are with the Aerospace Information Research Institute, Chinese Academy of Sciences, Beijing 100190 , China, and also with the Personalized Management of Chronic Respiratory Disease, Chinese Academy of Medical Sciences, Beijing 100190, China (email: wangpeng01@aircas.ac.cn; duld@aircas.ac.cn; chenxx@aircas.ac.cn ).

Chengyu Zhuang is with the Department of Orthopaedics, Shanghai Key Laboratory for Prevention and Treatment of Bone and Joint Diseases, Shanghai Institute of Traumatology and Orthopaedics, Ruijin Hospital, Shanghai Jiao Tong University School of Medicine, Shanghai 200025, China (E-mail: zhuangchengyu@msn.com)

Zhen fang is with the Aerospace Information Research Institute, Chinese Academy of Sciences, Beijing 100190, China, and with the University of Chinese Academy of Sciences, Beijing, 100049, China , and also with the Personalized Management of Chronic Respiratory Disease, Chinese Academy of Medical Sciences, Beijing 100190, China (e-mail: fangzhen@aircas.ac.cn). and daily life.

Index Terms-millimeter-wave FMCW radar, heartbeat monitoring, contactless sensing, template matching, dynamic programming

\section{INTRODUCTION}

Heartbeat is essential for the detection and diagnosis of cardiovascular conditions [1]. Accurate heartbeat monitoring is critical for clinical medicine [2], emotion recognition [3], sleep staging [4], etc. Additionally, dedicated devices are used for heartbeat monitoring, for example, photoplethysmography (PPG) [5] and electrocardiogram (ECG) [6]. These methods need standard clinical workflow. For more convenient monitoring, wearable devices are developed [7], [8]. However, when using these heartbeat monitor methods, user compliance problem exists [9]. If the user does not keep on wearing sensors, the heartbeat will not be monitored.

There have always been studies dedicated to making heartbeat monitoring unobtrusive. In 1877, Gordon found ballistocardiogram (BCG), a cardiogenic body vibration [10]. Various studies have obtained ballistocardiogram (BCG) with contactbased approach, like piezoelectric [11] sensor, hydraulic [12] sensor and etc. These sensors still need contact with the human body. Once the user leaves the specified position, the signal will not be obtained.

The past decade has witnessed tremendous innovation in using wireless signals to sense vital signs like respiration [13] and body temperature [14]. Due to its contactless nature, vital signs can be obtained when users are unconscious. Therefore, wireless monitoring technology can solve the user compliance problem well.

\section{A. Related Work and Limitations}

Recently, plenty of researches have demonstrate that heartbeat can be monitored through wireless approach including WIFI [15], acoustics [16], continuous wave (CW) radar [17][21], ultra-wideband radar (UWB) [22], frequency modulated continuous wave (FMCW) radar [3], [23]-[25] and etc.

These researches can be classified into two categories. One calculates the average heart rate within a time window; the other detects each individual heartbeat [26]. The former one divides the received signal into multiple time windows. For each time window, the average heart rate is estimated by spectrum analysis [23]. Some innovative algorithms use wavelet transform [24], non-negative matrix factorization [17], embedded ensemble empirical mode decomposition (EEMD) 
[27], etc. to recover the heartbeat harmonics under the interference of respiration and body movements, intend to obtain a more accurate estimate.

Compared with average heart rate monitoring, there are a few studies on non-contact IBI estimation. After in-depth research, we find the primary reason is the sensitivity to interference. As far as we know, although using different signals, all contactless approaches sense heartbeat through measuring the displacement of specific surfaces on the human body. The displacement caused by the heartbeat is tiny and susceptible to body movement. Even when users stay static, the heartbeat could be overwhelmed by respiration.

Some confirmatory studies require subjects to hold their breath, observing that each heartbeat cycle in the heartbeat signal has a similar shape [28], [29]. These methods cannot be used for long-term monitoring.

Will et al. [19] make the antenna focus on the specific parts of the human body where the vibration caused by heartbeat is most observable, for example, the carotid artery and the venous. Furthermore, the obtained heartbeat signal is similar to a sphygmogram. However, the distance between radar and subjects must be small (less than $20 \mathrm{~cm}$ ), and participants must stay static without changing posture and position.

Systems proposed in [3] and [16], respectively, can estimate IBI accurately in different posture and posture. Whereas the computational complexity of their IBI estimation algorithm is incredibly high. Their algorithm requires high CPU performance, and real-time calculations cannot be achieved on a low-cost CPU.

Wang et al. [25] proposed a HRV monitoring system. With low complexity, this system can estimate IBI and HRV parameters in different postures and postures. However, their IBI estimation method is based on sequentially detect peaks. This method is not robust enough when there is a large variation of heartbeat shape, which is discussed in section VII.

\section{B. Our Proposal}

Motivated by existing work, in this paper, we present RFHeartbeat, a heartbeat monitoring system based on millimeterwave FMCW radar, which enables contactless, long-term monitoring. Our approach is robust to changes in posture and position and can deal with body movement. With high performance, RF-Heartbeat achieves distinctly low computational complexity.

Among all wireless technologies, the unique advantages of millimeter-wave frequency modulated continuous wave (FMCW) radar caught our attention: 1) Millimeter-level wavelength makes it has a high sensitivity for detecting small displacements. 2) FMCW Radar can divide reflected signals into different range bins. Therefore, it can isolate noise from areas we do not interest in. For example, small movements of legs do not affect the radar to obtain the reflected signal from the surface of the chest cavity. In addition, for FMCW radar, using beamforming and the human body location algorithm, the single-person heartbeat monitoring algorithm can be extended to suit the multi-person scenario. Thus, the millimeter-wave FMCW radar is selected to carry out our work.
There are still plenty of challenges in monitoring heartbeat based on millimeter-wave FMCW radar. First, body movements of different amplitudes may occur at any time, and it is not easy to ensure the algorithm's robustness. Second, with relatively small range bins, millimeter-wave FMCW radar can isolate body movements and respiratory from other range bins. Nevertheless, it is harder to select the range bin for extracting heartbeat. Most previous works selected range bins based on reflection power. However, the range bin from the strongest reflection may not coincide with the most obvious heartbeat but rather a different range bin on the chest or back. Third, unlike ECG and BCG, for heartbeat signal acquired by radar, there are no characteristic points like $\mathrm{R}$ peak and $\mathrm{J}$ peak defined by experts to identify each heartbeat cycle. Therefore, IBI estimation is relatively difficult.

The high-frequency part of the heartbeat signal is found to be almost unaffected by the respiration signal. In order to retain its high-frequency components, the signal is preprocessed by a bandpass filter.

For the second challenge, our solution is to pinpoint the signal segments with body movement and discard them. We let RF-Heartbeat contain a body movement detection method, which introduced body movement index (BMI) for quantifying body movement amplitude. BMI is sensitive to body movement. With this design, RF-Heartbeat surpasses previous work [23] by avoiding discarding signal segments without body movement.

To overcome the second challenge, we propose a robust range bin selection algorithm. An Index reflecting SNR based on Pearson correlation coefficient (PCCs) [30] is defined. According to this index, the optimal range bin can be automatically selected. When large distortion caused by body movement exist, PCCs might judge tiny heartbeat signal as noise. However, with a precise body movement detection method, the SNR index would not be affected. This algorithm significantly improves the robustness of RF-Heartbeat. When a user is in different postures and positions, the optimal range bin can be selected adaptively. Furthermore, with this design, the proportion of heartbeat signals increased significantly relative to respiration. Therefore, it is no more difficult to eliminate respiratory interference.

For the third challenge, RF-Heartbeat uses template matching for heartbeat segmentation. The difficulty of template matching is the choice of template. When people are in different postures and positions, the shape of each heartbeat is different. Therefore, RF-Heartbeat uses the affinity propagation (AP) clustering algorithm [31], taking one heartbeat signal series as input and learning a template specific to each series. The template matching algorithm is based on a hypothesis: Each heartbeat has a similar shape. However, in long-term contactless monitoring, that hypothesis is not supported [32]. To make heartbeat segmentation more robust. We design a global optimization model based on the generated template, heartbeat signal, and prior knowledge of HRV. Dynamic programming [33] is used to solve this model to obtain the global optimal heartbeat segmentation.

The main contributions of our work can be summarized as follows: 
- A precise body movement detection algorithm is proposed.

- An adaptive and robust range bin selection strategy is proposed for extracting high-quality heartbeat signals when users are in different positions and postures.

- We use dynamic programming to enhance the robustness of the template matching algorithm and propose a heartbeat segmentation algorithm based on global optimization. In long-term monitoring, this algorithm can robustly estimate IBI and keep high accuracy.

RF-Heartbeat relies on low-cost millimeter-wave FMCW radar. Millimeter-wave signals are able to traverse clothes and quilt. Such an unobtrusive approach can monitor cardiovascular conditions in clinical space and daily life. Our system can provide early warning of potential problems and perceive cardiovascular emergencies like cardiac arrest in time. These abilities could be especially beneficial to high-risk populations, like the elderly, neonates, and patients with arrhythmia.

Furthermore, RF-Heartbeat has excellent application prospects in the clinical space. For example, currently, COVID-19 has become a global epidemic. In the treatment of infectious diseases like COVID-19, monitoring vital signs is significant, and wireless monitoring will decrease the cross-infection risk.

\section{Heartbeat Signal Capture}

In this section, we describe the principle of millimeter-wave FMCW radar, parameters of the radar used in RF-Heartbeat, and how RF-Heartbeat extracts heartbeat signal.

Several related studies described the principle of FMCW radar [3], [23], [34]. For knowing how FMCW works in detail, we refer the reader to [34]. Briefly, chirps with linearincreasing frequency are transmitted periodically given by

$$
S_{T}(t)=A_{t} \cos \left(2 \pi f_{c} t+\pi \frac{B}{T_{c}} t^{2}\right)
$$

where $A_{T}$ is the magnitude of chirp. $f_{c}$ is the starting frequency of a chirp. $B$ and $T_{c}$ are the bandwidth and duration of a chirp, respectively. The signals reflected from multiple targets in the radar field of view are received, and each reflected signal is a scaled and time-shifted version of the transmitted signal. Through mixing with the transmitted signal $S_{T}(t)$, the output IF signal is composed of multiple harmonics, given by

$$
s_{I F}(t)=\sum_{n} A_{n} \cos \left(2 \pi f_{n} t+\phi_{n}(t)\right) .
$$

The radar field of view is divided into multiple range bins by using FFT transformation. $n$ is the range bin index in (2). Let $d_{n}$ denote the distance between radar and reflector in $n$-th range bin, then

$$
f_{n}=\frac{2 f_{c} B d_{n}}{c}
$$

is defined as frequency corresponding to the $n$-th range bin, and $c$ is the speed of light. $A_{n}, \phi_{n}(t)$ are the amplitude and phase of the reflected signal from the $n$-th range bin, respectively. When position of the reflector in the $n$-th range bin changes slightly, that is, $D_{n}(t)=d_{n}+\delta_{n}(t)$, and this slight

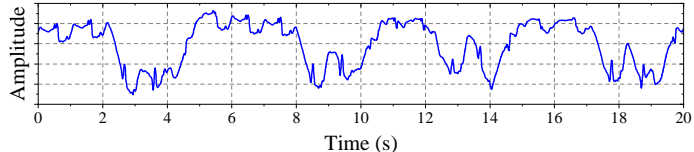

(a) Phase signal

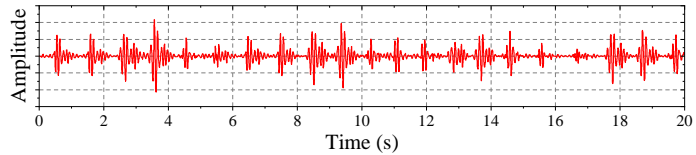

(b) Filtered phase signal

Fig. 1. Example of heartbeat signal extracting (a) Phase signal of appropriate $n$-th range bin, $\phi_{n}(t)$ (b) Signal obtained by filtering $\phi_{n}(t)$ with $8-24 \mathrm{~Hz}$ Butterworth bandpass filter.

displacement $\delta_{n}(t)$ do not cause reflector to move beyond the range bin, and $\phi_{n}(t)$ is modulated by $\delta_{n}(t)$. These modulation is expressed as

$$
\phi_{n}(t)=2 \pi \frac{f_{c}\left(D_{n}+\delta_{n}(t)\right)}{c} .
$$

There are static objects like walls and furniture in the real environment, and reflections from these objects need to be eliminated. Let $S_{I F}^{k}(t)$ be the $i$-th IF signal received by radar. For any $k$, the signal after static objects elimination is defined as

$$
\tilde{S}_{I F}^{k}(t)=S_{I F}^{k}(t)-\frac{1}{2 N} \sum_{i=k-N-1}^{k+N} S_{I F}^{i}(t) .
$$

Heartbeat causes displacement of some specific reflective human body surface. If focusing on the range bins covering these surfaces, the heartbeat signal can be extracted from the phase signals.

As mentioned before, the heartbeat signal is often overwhelmed by the respiration signal. We use an $8-24 \mathrm{~Hz}$ Butterworth bandpass filter to remove the respiration signal and low-frequency components to eliminate this interference. As shown in Fig. 1, in the high-frequency components of the heartbeat signal, each heartbeat still has a similar shape.

Different from some previous researches [16], [25], which use original and complicated methods to remove respiratory interference, we adopt a Butterworth filter. The main reason is that we adopt a smaller range bin $(3.75 \mathrm{~cm})$. With a smaller range bin, RF-Heartbeat can select the range bin with the largest amplitude of cardiogenic body vibration and isolate respiratory interference from other areas. Therefore, in some range bins, the heartbeat component is more obvious, as shown in Fig. 1(a), and respiratory interference can be eliminated purely using the Butterworth filter. Whereas, with this design, the difficulty is the selection of range bin, which is elaborated in section IV.

\section{Body Movement Detection}

A previous study proposed the body movement index (BMI) [35], which was used to quantify the body movement amplitude of subjects based on radar signals. We introduce this index 
for body movement detection and modify some parameters to make it more sensitive to changes in body movement amplitude, as shown in Fig. 2. Let $x$ denote the heartbeat signal obtained through the process mentioned before, and the sampling rate is $250 \mathrm{~Hz}$. $\left\{x[t]_{t=i-N}^{i+N-1}\right\}$ is the $4 \mathrm{~s}$ signal segment $(N=500)$ near the $i$-th sampling point. $A[i]$ is defined as the integral of the absolute value of this signal segment:

$$
A[i]=\sum_{t=i-N}^{i+N-1}|x[t]| .
$$

Body movement index can be expressed as

$$
B M I[n]=\sum_{i=n-75}^{n+75}\left(A[i]-A_{\min }[i]\right),
$$

where $A_{\min }[i]$ is the minimum value of $A[i]$ within 10 seconds near the $i$-th point.

Different positions and different postures could cause different amplitudes of the signal $x[t]$. Taking these into consideration, we adopt an adaptive body motion detection strategy. First, set a sliding time window with a size of $2 \mathrm{~s}$ and a step size of $1 \mathrm{~s}$. We calculate the sum of the body movement index in each time window, which is called the accumulative body movement index (ABMI). Then, we use statistical analysis, box plot [36], to calculate the distribution of ABMI across all time windows and extracts the first quartile $\left(Q_{1}\right)$ and third quartile $\left(Q_{3}\right)$. Based on these values, a threshold $T_{h}$ is defined:

$$
T_{h}=Q_{3}+1.5 \times\left(Q_{3}-Q_{1}\right) .
$$

Then, if ABMI of any time window exceeds this threshold, RF-Heartbeat will discard it. Mask $m$ is defined to describe whether body movement occurs. If our method judges that body movement occurs in time point $t, m[t]$ will be set to 1 , else $m[t]$ will be set to 0 , as shown in Fig. 2. $m[t]$ is a significant index for following sections.

\section{Optimal Range Bin Selection}

Different from previous work, millimeter-wave FMCW radar can divide the radar field of view into several centimeterlevel range bins. Different parts of the human body are located in different range bins. Relevant studies have shown that specific areas of the thoracic cavity and the carotid artery are the best choices for detecting vibrations caused by heart activity [37]. As the position and posture change, the distance between these specific positions and the radar is uncertain. When the user is in some postures, the vibration of a specific location cannot be captured by radar. For example, when users are prone, radar cannot obtain the reflection of the chest cavity. Therefore, we propose an algorithm to automatically select the optimal range bin for extracting the heartbeat signal.

The transmitter is configured to periodically send two consecutive chirps, the interval between the two chirps is very short $(100 \mu s)$. Every two chirps are called a frame. In each frame, after the $l$-th chirp sampled and FFT transformed, the phase signal of the $n$-th range bin is $\phi_{n}^{l}$. After filtering $\phi_{n}^{1}$ and $\phi_{n}^{2}$, two sets of heartbeat signals $x_{n}^{1}$ and $x_{n}^{2}$ are obtained. $x_{n}^{1}$ and $x_{n}^{2}$ consists of random noise, body movement noise,
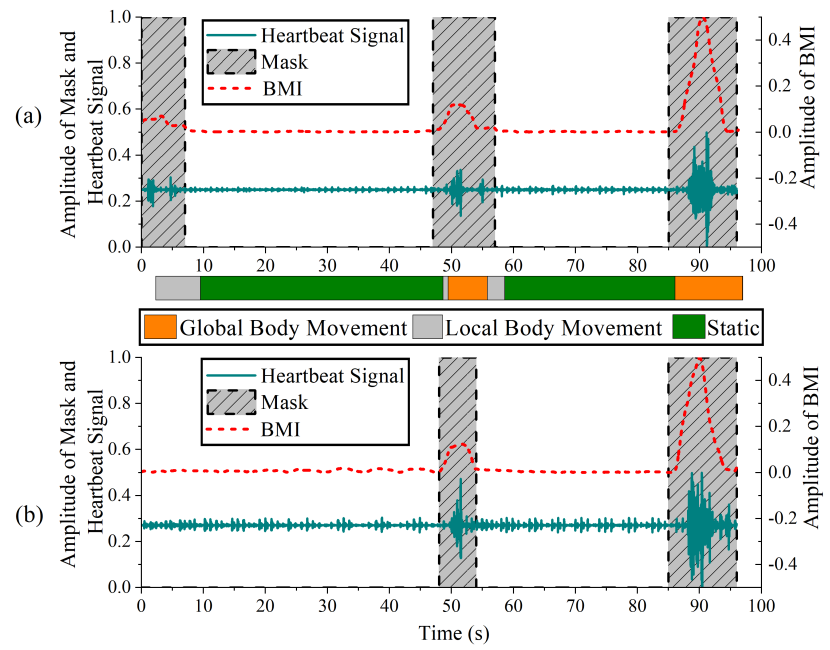

Fig. 2. Example of BMI for reflecting amplitude of body movement, and mask for indicating whether body movement occurs. In Global body movement time windows, body movements are detected in multiple adjacent range bins. In local body movement windows, body movements occur in some range bins. (a) The heartbeat Signal, BMI, and mask in one range bin; (b) The heartbeat Signal, BMI, and mask measured simultaneously as (a), in another adjacent range bin.

and heartbeat signal. When an obvious heartbeat signal can be extracted in the $n$-th range bin, there is a strong correlation between $x_{n}^{1}$ and $x_{n}^{2}$. On the contrary, when the heartbeat signal is less observable, the proportion of random noise is larger, and the correlation between $x_{n}^{1}$ and $x_{n}^{2}$ is smaller. PCCs $\rho$ is selected to quantify the correlation between $x_{n}^{1}$ and $x_{n}^{2}$, expressed as

$$
\rho\left(x_{n}^{1}, x_{n}^{2}\right)=\frac{\sum_{t}\left(x_{n}^{1}-\overline{x_{n}^{1}}\right)\left(x_{n}^{2}-\overline{x_{n}^{2}}\right)}{\sqrt{\sum_{t}\left(x_{n}^{1}-\overline{x_{n}^{1}}\right)^{2}} \sqrt{\sum_{t}\left(x_{n}^{2}-\overline{x_{n}^{2}}\right)^{2}}}
$$

where $\overline{x_{n}^{1}}$ and $\overline{x_{n}^{2}}$ are the averages of $x_{n}^{1}$ and $x_{n}^{2}$, respectively.

When body movement happens, since the heartbeat signal could be overwhelmed by the body movement noise, $\rho$ could be assigned greater weight to the body movement noise, which cannot reflect the SNR of the heartbeat signal. Therefore, our algorithm only selects the signal segment without body movement to calculate $\rho$.

Body movement might cause changes in distance from radar and posture. Based on realizing precise detection of the period with body movement, our algorithm can dynamically adjust the optimal range bin. The range bin $n$ with the largest amplitude of $x_{n}^{1}$ is counted for the acquired radar signal in each slow time point. The range bin with the largest frequency in the statistic result is denoted as $r$. For the $l$-th chirp, the heartbeat signal of $2 m+1$ range bins near $r$ is $x_{r-m}^{l}, \ldots, x_{r}^{l}, \ldots, x_{r+m}^{l}$. For the signal of each distance unit, calculate the time window with body movements. We define

$$
P_{i}=\left\{p_{i}^{1}, p_{i}^{2}, \ldots, p_{i}^{n_{i}}\right\}
$$

as the body movement time window set detected in the $i$ th $(i \in\{r-m, r-m+1, \ldots, r+m\})$ range bin. If body movement is detected in $2 m+1$ range bins within the same 


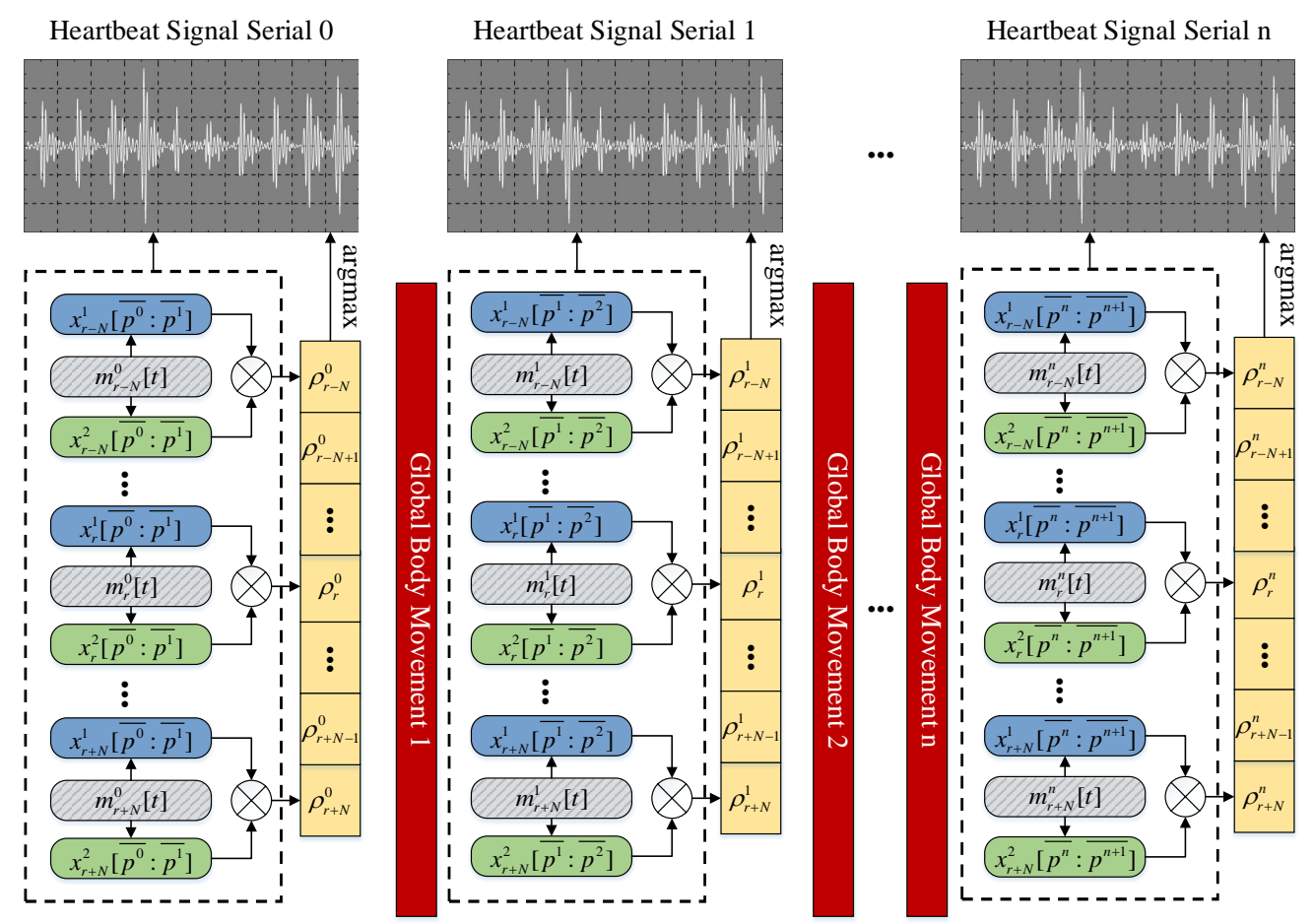

Fig. 3. The overall flow of proposed method for optimal range bin selection, where $\rho_{r}^{n}=\rho\left(x_{r}^{1}\left[\overline{p^{n}}: \overline{p^{n+1}}\right] \cdot\left(1-m_{r}^{n}\right), x_{r}^{2}\left[\overline{p^{n}}: \overline{p^{n+1}}\right] \cdot\left(1-m_{r}^{n}\right)\right)$.

time window, it means that there might be changes in posture or position within that time window. We define this body movement as a global body movement, as shown in Fig. 2. Before and after global body motion, our algorithm needs to consider the change of the optimal range bin. Global body movement time window set is defined as

$$
P=\left\{p^{1}, p^{2}, \ldots, p^{n}\right\}=P_{R-M} \cap P_{R-M+1} \cap \ldots \cap P_{R+M}
$$

which is the union of the body movement time window sets detected in each range bin. Global body movement time point set is given by

$$
\bar{P}=\left\{\overline{p^{0}}, \overline{p^{1}}, \ldots, \overline{p^{n}}, \overline{p^{n+1}}\right\}
$$

where $\overline{p^{i}}(i \in\{1,2, \ldots, n\})$ is the midpoint of $i$-th global body movement time window $p^{i} \cdot \overline{p^{0}}$ and $\overline{p^{n+1}}$ are the first and last sampling points of heartbeat signal, respectively. Local body movement time window set of the $i$-th range bin is defined as

$$
Q_{i}=\left\{q_{i}^{1}, q_{i}^{2}, \ldots, q_{i}^{n_{i}-n}\right\}=\complement_{P_{i}} P
$$

which is the absolute complement of $P$ in $P_{i}$, as shown in Fig. 2. Let $x_{r}^{l}\left[\overline{p^{i}}: \overline{p^{i+1}}\right]$ denote the segment of $x_{r}^{l}$ between global body movement time point $\overline{p^{i}}$ to $\overline{p^{i+1}}$. Based on $P, \bar{P}$ and $Q_{i}$, The mask of $i$-th range bin is constructed given by

$$
M_{i}=\left\{m_{i}^{o}, m_{i}^{1}, \ldots, m_{i}^{n}\right\},
$$

where $m_{i}^{k}[t]$ is the mask of $x_{i}^{1}\left[\overline{p^{k}}: \overline{p^{k+1}}\right]$.

The optimal range bin set $R^{*}=\left\{r_{0}^{*}, r_{1}^{*}, \ldots, r_{n}^{*}\right\}$ can be solved using following optimization strategy

$$
R^{*}=\underset{\left\{r_{0}^{*}, r_{1}^{*}, \ldots, r_{n}^{*}\right\}}{\arg \min } \sum_{i=0}^{n} \frac{\rho^{i} \sum_{t}\left(1-m_{r_{i}}^{i}[t]\right)}{\overline{p^{i+1}}-\overline{p^{i}}}
$$

where

$$
\begin{aligned}
\rho^{i}= & \rho\left(x_{r_{i}}^{1}\left[\overline{p^{i}}: \overline{p^{i+1}}\right] \cdot\left(1-m_{r_{i}}^{i}\right),\right. \\
& \left.x_{r_{i}}^{2}\left[\overline{p^{i}}: \overline{p^{i+1}}\right] \cdot\left(1-m_{r_{i}}^{i}\right)\right) .
\end{aligned}
$$

Since body movement noise significantly impacts $\rho$, the body movement mask is introduced. When calculating $\rho$, for better reflecting the SNR of the heartbeat signal, this mask sets the intensity of signal segment during the period when body movement happens to 0 . The coverage index

$$
\frac{\sum_{t}\left(1-m_{r_{i}}^{i}[t]\right)}{\overline{p^{i+1}}-\overline{p^{i}}}
$$

is defined as the ratio of the time without body movement to the total time during the period from $\overline{p^{i}}$ to $\overline{p^{i+1}}$. Our algorithm achieves a trade-off. While choosing the range bin with the largest SNR, RF-Heartbeat tends to choose the range bin with less local body movements. This trade-off can improve the time coverage of our algorithm.

Our optimal range bin algorithm is summarized as an overall flow, as shown in Fig. 3.

\section{IBI Estimation Algorithm}

Based on the body movement detection and optimal range bin selection algorithm mentioned before, the radar signals received are processed into multiple heartbeat signal series without body movement. To simplify the description, we only introduce the heart rate estimation process of a single heartbeat signal series without body movement. For a single heartbeat signal series, an IBI estimation algorithm based on template matching is established. Our algorithm is based on an intuition: 
(a)

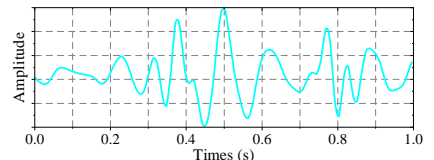

(b)

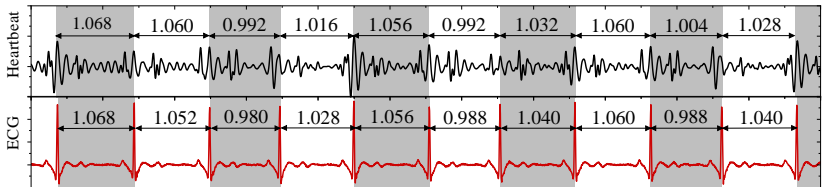

(c)

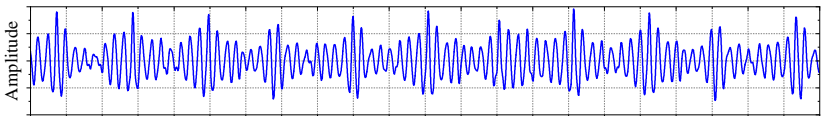

(d)

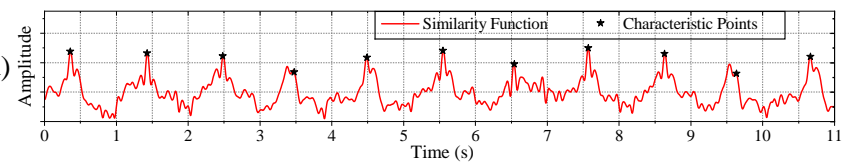

Fig. 4. Input, output and some important intermediate results of our Heartbeat Segmentation Algorithm. (a) Generated heartbeat template. (b) Heartbeat segmentation result. (c) correlation coefficient function Corr. (d) Similarity function $e$ and characteristic points $\left\{\tau_{1}, \tau_{2}, \cdots, \tau_{n}\right\}$

Although different position and posture could cause diversity in the shape of a single heartbeat cycle, in a heartbeat signal without body movement, even if the length of each heartbeat changes due to heart rate variability, the shape of each single heartbeat cycle is similar.

\section{A. Template generation}

Compared with ECG, the heartbeat signal received by radar does not have a standard shape. So the selection of the template is more complicated. Our thought is to truncate a representative segment from the heartbeat signal as the template. If the correct template is selected, enough signal segments are similar to the template. Based on the above ideas, a template generation algorithm based on clustering is proposed.

$x$ is defined as a heartbeat signal series without any body movement. All maximum points in $x$ are marked. Two hundred sampling points before and after each maximum point are intercepted to obtain a segment of $0.8 \mathrm{~s}$. These signal segments constitute the template learning sample set $A=\left\{a_{i}, a_{2}, \ldots, a_{n}\right\}$. For declining the influence of amplitude changes, different from other works using Euclidean distance, our algorithm uses PCCs $\rho$ defined in (7) to quantify the similarity between heartbeat signal segments and template. Based on $\rho$, the similarity matrix $S$ is defined by

$$
S=\left[\begin{array}{cccc}
M(S) & s\left(a_{1}, a_{2}\right) & \cdots & s\left(a_{1}, a_{n}\right) \\
s\left(a_{2}, a_{1}\right) & M(S) & \cdots & s\left(a_{2}, a_{n}\right) \\
\vdots & \vdots & \ddots & \vdots \\
s\left(a_{n}, a_{1}\right) & s\left(a_{n}, a_{2}\right) & \cdots & M(S)
\end{array}\right]
$$

where

$$
s\left(a_{i}, a_{j}\right)=\rho\left(a_{i}, a_{j}\right)-1, \quad i, j \in\{1,2, \cdots, n\}
$$

and $M(S)$ is the median of similarity matrix $S$.
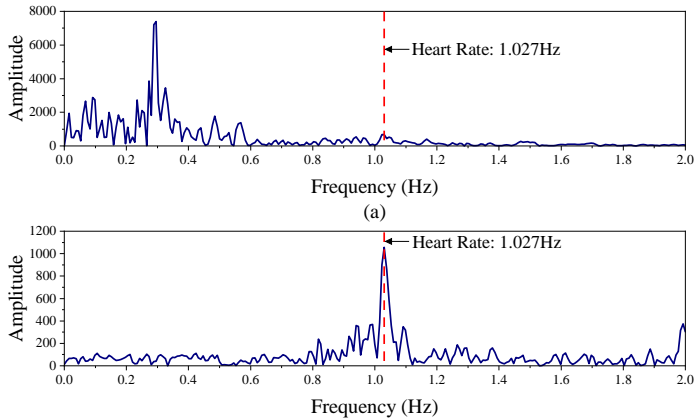

(b)

Fig. 5. The spectrum of phase signal and similarity function (average heart rate of synchronized ECG is $1.027 \mathrm{~Hz}$ ). (a) The spectrum of phase signal of optimal range bin (b) Spectrum of similarity function $e$.

Affinity propagation (AP) clustering algorithm is selected for template generation, using similarity matrix $S$ as input. The cluster center of each class of AP clustering algorithm outputs is a signal segment. To understand the AP clustering algorithm in detail, we refer [31] to readers. The cluster center of the class with the most significant number of samples is selected as the template.

\section{B. Heartbeat Segmentation Algorithm}

A template $T$ specific to a heartbeat signal series can be obtained through the method mentioned above, as shown in Fig. 5(a). We use a sliding time window with the same length as the template, taking 1 sample point as the step size. Calculating $\rho$ between the template and the signal in each sliding time window with size $2 N(N=125)$, correlation coefficient function $\operatorname{Corr}$ can be obtained given by

$$
\operatorname{Corr}[t]=\rho(x[t-N: t+N-1], T)
$$

where $x[t-N: t+N-1]$ is the segment of heartbeat signal $x$ between $t-N$ and $t+N-1$, shown in Fig. 4(c). The more similar the heartbeat signal segment to the template, the higher $\operatorname{Corr}[t]$. However, because the range of PCCs is $[-1,1]$, Corr $[t]$ fluctuates repeatedly between positive and negative values. We perform the Hilbert transform on Corr to obtain the envelope $e$, called similarity function, shown in Fig. 4(d). After this processing, the function qualifying similarity between template and heartbeat signal within each time window is smoother. Meanwhile, $e$ is always positive. When the heartbeat signal in a time window is highly similar to the template, a positive peak always appears in $e$. Signal in a time window is more similar to the template, the higher $|\operatorname{Corr}[t]|$ is. However, since the value range of PCCs is $[-1,1]$, Corr fluctuates repeated.

In previous studies on IBI estimation, after obtaining a function similar to $e$, most researchers adopted a strategy of sequentially detecting peaks [21], [38], [39]. Unfortunately, many factors cause variation in heartbeat shape, for example, respiratory [32]. When there are some heartbeats with a significant difference in shape from others, for similarity function $e$, a considerable peak might not be detected in these heartbeats.

In this case, the algorithm that sequentially detects peaks has poor robustness. If this algorithm is used, once the IBI 
is misestimated at one heartbeat, it will affect estimation accuracy after that heartbeat. To make IBI estimation robust and precise, we propose a global optimization strategy.

Based on the intuition that the amplitude change of $e$ has the same periodicity as the heartbeat. After performing FFT on $e$, and taking the frequency $f_{0}$ corresponding to the spectrum's peak, the average heart rate of that heartbeat signal series is estimated. Compared with the phase signal of the optimal range bin, $e$ has a more obvious peak in the reasonable heartbeat frequency range, as shown in Fig. 5. As the output of algorithm, the set of characteristic points of $x$ is $T=\left\{\tau_{1}, \tau_{2}, \cdots, \tau_{n}\right\}$, then

$$
l_{i}=t_{\tau+1}-\tau_{i}, \quad i \in\{1,2, \cdots, n-1\}
$$

is the length of $i$-th heartbeat. Theoretically, when there is no distortion in the heartbeat signal, $T$ is the peak points set of $e$. Meanwhile, the difference between the $l_{i}$ and $l_{i+1}$, lengths of two adjacent heartbeats, should be within a reasonable range; the difference between $l_{i}$ and the average heartbeat length $\frac{1}{f_{0}}$ should also be within a reasonable range. In summary, we define an optimization model:

$$
\begin{aligned}
T^{*}= & \underset{T}{\arg \max } \sum_{\tau_{i} \in T} e\left[\tau_{i}\right]-\lambda_{1} \sum_{\tau_{i}, \tau_{i+1} \in T} g_{1}\left(\tau_{i}, \tau_{i+1}\right) \\
& -\lambda_{2} \sum_{\tau_{i}, \tau_{i+1}, \tau_{i+2} \in T} g_{1}\left(\tau_{i}, \tau_{i+1}, \tau_{i+2}\right) \\
& \text { s.t. } \quad \frac{1}{f_{0}}-3 \sigma_{s}<\tau_{i+1}-\tau_{i}<\frac{1}{f_{0}}+3 \sigma_{s} .
\end{aligned}
$$

We define the two loss terms in (21)

$$
g_{1}\left(\tau_{i}, \tau_{i+1}\right)=\frac{1}{\sqrt{2 \pi} \sigma_{s}}-g\left(\tau_{i+1}-\tau_{i} \mid \frac{1}{f_{0}}, \sigma_{s}\right)
$$

and

$$
g_{1}\left(\tau_{i}, \tau_{i+1}, \tau_{i+2}\right)=\frac{1}{\sqrt{2 \pi} \sigma_{r}}-g\left(\tau_{i+2}-2 \tau_{i+1}+\tau_{i} \mid 0, \sigma_{r}\right)
$$

as heart rate variety (HRV) loss, where $g(x \mid \mu, \sigma)$ is a Gaussian function with average $\mu$ and variance $\sigma^{2}, \sigma_{s} \approx 180$ $\mathrm{ms}$, is the upper bound of the standard deviation of IBI (SDNN) under normal conditions, and $\sigma_{r} \approx 54 \mathrm{~ms}$ is the upper bound of the root mean square of the difference between lengths of two adjacent heartbeats (RMSSD) under normal circumstances. We assume that for the entire user group, IBI and its difference between the length of two adjacent heartbeats obeys a Gaussian distribution, and the constraint condition of (21) can cover almost all situations.

Through dynamic programming (DP) algorithm, the optimization model in (21) can be solved. $N$ denoted as the length of the heartbeat signal series, Algorithm 1 describes the solution process of (21) in detail.

\section{RESULT}

In this section, the accuracy and robustness of RF-Heartbeat are evaluated.

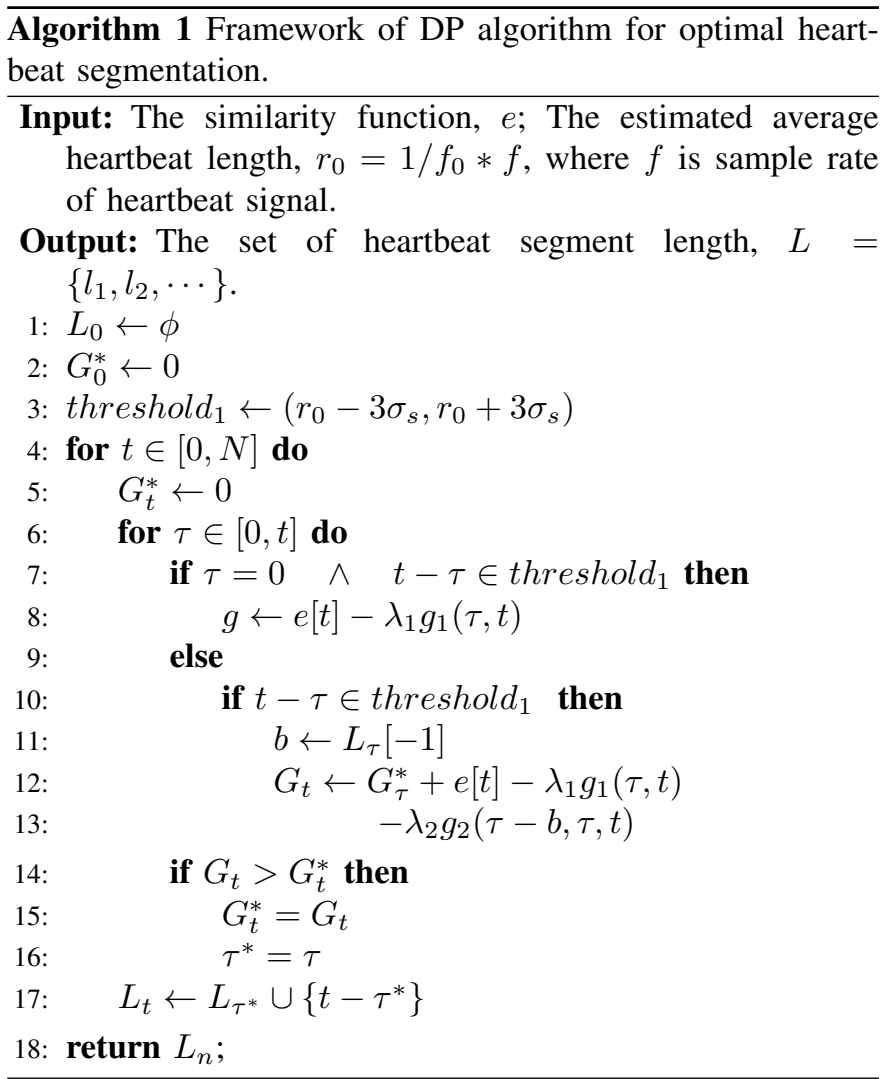

\section{A. Experiment Setup}

Methodology: As described in section II.B, we set the parameters of millimeter-wave FMCW Radar in our experiment. The DCA1000EVM is a capture card for interfacing with Texas Instruments 77GHz xWR1xxx EVM, including AWR1642 EVM. After connecting AWR1642 with the DCA1000EVM, the raw IF signal can be streamed to a computer through Ethernet. Our algorithm runs on a computer, takes the raw IF signal as input, and outputs heartbeat signal and IBI estimation. In order to evaluate the performance of RF-Heartbeat, we use SOMNOtouch PSG to record I lead ECG and use the algorithm described in [40] to detect $R$ peak to obtain the ground truth of HRV. The example of wearing SOMNOtouch Polysomnography (PSG) is shown in Fig6. (a) and Fig. 6(b). In addition, SOMNOtouch PSG can record the posture of subjects, the amplitude of body movements. Besides, snoring events during sleep can be detected with a nasal cannula, as shown in Fig. 7. These labels are used to evaluate the robustness of RF-Heartbeat, which is elaborated in subsequent sections.

Participants: In this experiment, ten volunteers participated, six males and four females, aged between 20 and 31 . Participants wore their daily clothes with different materials, such as T-shirts, blouses, etc.

Experiment environment: Our experiments were performed in an office with standard furniture and other wireless devices like WiFi, Bluetooth, etc. During experiments, participants were asked to lie on a bed within $80-100 \mathrm{~cm}$ from the radar, as shown in Fig. 6(c). Thus, their postures can be automatically recorded using SOMNOtouch PSG. During the 


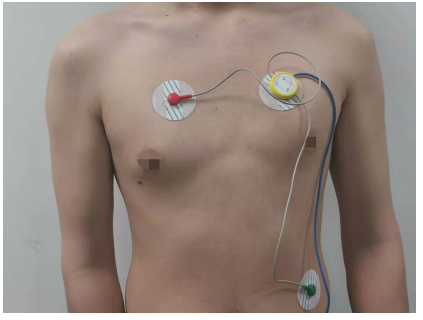

(a) ECG leads

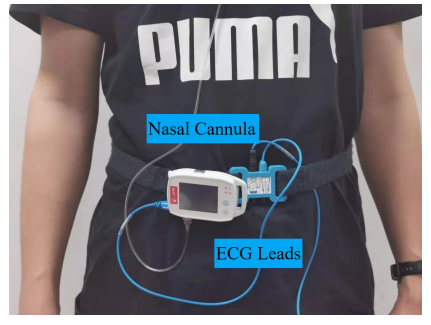

(b) SOMNOtouch PSG

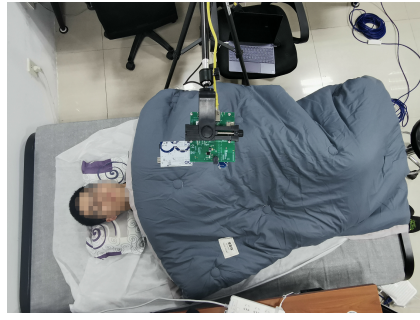

(c) Heartbeat signal capturing

Fig. 6. Setup of our experiment for evaluating the performance of the proposed algorithm. (a)ECG leads connected to SOMNOtouch PSG. (b) Example of wearing SOMNOtouch PSG for providing ground truth and significant labels. (c) Equipment deployment for capturing heartbeat signal.

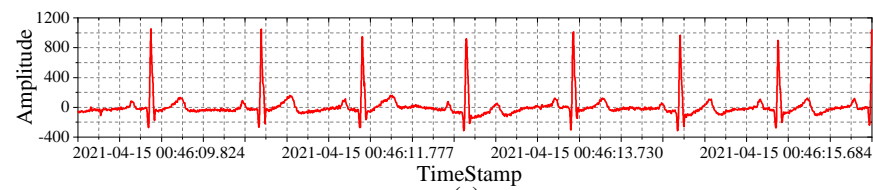

(a)

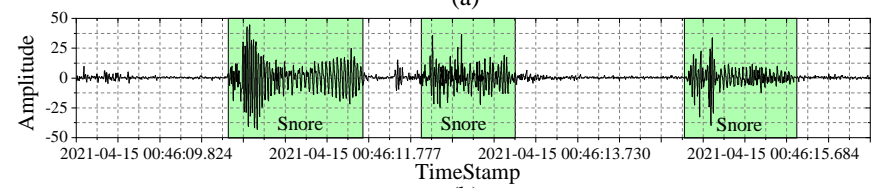

(b)

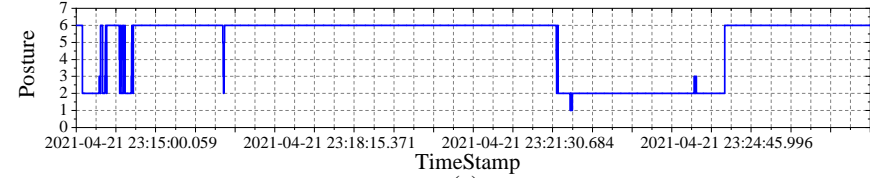

(c)

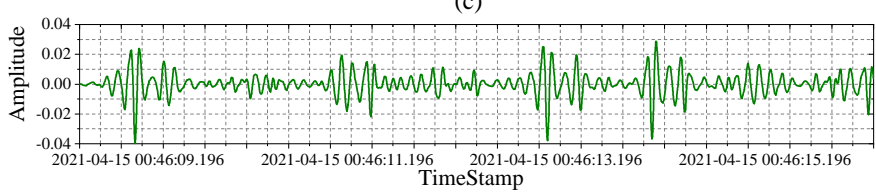

(d)

Fig. 7. Ground truth, labels, and heartbeat signal recorded by SOMNOtouch PSG. (a) I lead ECG in the obtained ground truth of IBI. (b) The pressure snore recorded with a nasal cannula. By analyzing pressure snore, The PSG we use can detect snore events. (c) The posture record. When the value is 1 , 3,4 , and 6 , the posture of the subject is lying on the stomach, lying on the left, lying on the right, and lying on the back, respectively. When the value is 2 and 5, the subjects are sitting or standing. (d) Collected heartbeat signal using radar.

data recording process, participants breathed normally, lay in a comfortable posture for them, and could change their position at will. In the experiment setup mentioned before, al A total of 72 hours and 56 minutes of radar data were recorded. The time of the shortest record is 5 hours and 52 minutes, and the longest is 8 hours and 26 minutes. In each record, at least 4 hours of data were collected when participants were asleep. During sleep, The participant's movements, posture changes, and snoring were not under control.

Evaluation indexes: The following indexes are defined for evaluating RF-Heartbeat:

- IBI error: Because the R peak of ECG seldom coincide with the characteristic points of the heartbeat signal, for the $i$-th heartbeat estimate, let $R R_{i}$ denote the RR interval with the largest overlap in time with $l_{i}$, and the $I B I$ error of the $i$-th heartbeat is defined as

$$
I B I_{i}=\left|R R_{i}-l_{i}\right|
$$

The unit is number of millisecond (ms).

- HR error: This error is defined as the absolute value of the difference between estimated beat-to-beat heart rate and ground truth, given by

$$
H R_{i}=60 \times\left|\frac{1}{R R_{i}}-\frac{1}{l_{i}}\right| .
$$

The unit is number of heartbeats per minute (bpm).

\section{B. Overall Performance of RF-Heartbeat}

For the collected data mentioned in VI.A, under $91.22 \%$ time coverage, the median IBI error of RF-Heartbeat is 12 $\mathrm{ms}$, and the median HR error is $0.655 \mathrm{bpm}$.

We took the heartbeat signal series without body movement as input, and compared RF-Heartbeat with ALG1 and ALG2, proposed in [3], and [25], respectively. ALG1 is also based on template matching and dynamic programming, to the best of our knowledge, is the most accurate algorithm for wireless HRV estimation. ALG2 is based on template matching, which sequentially searches for the peak value of the correlation coefficient between the template and the heartbeat signal.

Fig. 8(a), Fig. 8(b) plot the cumulative distribution function (CDF) of estimation error and IBI error of our algorithm and the other two algorithms. The IBI median errors of RFHeartbeat, ALG1, and ALG2 are $12 \mathrm{~ms}, 16 \mathrm{~ms}, 16 \mathrm{~ms}$, respectively. Compared with the other two algorithms, the time complexity of RF-Heartbeat is evaluated. The time consumption of running three algorithms in the Intel(R) Core(TM) i7-8750H CPU is shown in Fig. 8(c). Intend to reflect the distribution of HRV estimation results in more detail, Fig. 8(d) and Fig. 8(e) show the Bland-Altman diagram of HRV estimation and ground truth.

Results show that the performance of RF-Heartbeat is better than ALG1, and time complexity is much lower than the latter.

In RF-Heartbeat, the template is regarded as a representative segment of the heartbeat signal. Like the J peak of the BCG signal, the pattern similar to generated template appears at the same position in each heartbeat cycle. The length of the generated template does not need to be equal to the whole heartbeat cycle. Therefore, unlike ALG1, which uses linear warping to adjust the template's length dynamically, we use the template with a constant length. Even if the template and 


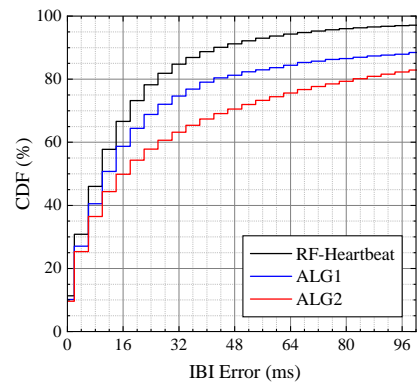

(a) CDF of IBI error

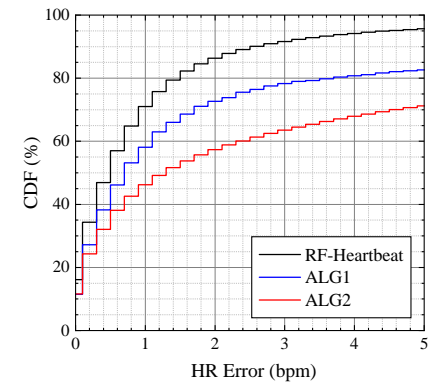

(b) $\mathrm{CDF}$ of HR error

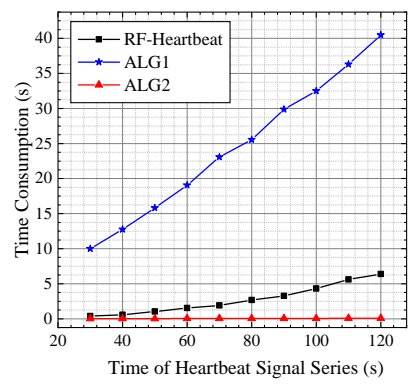

(c) Time consumption comparison

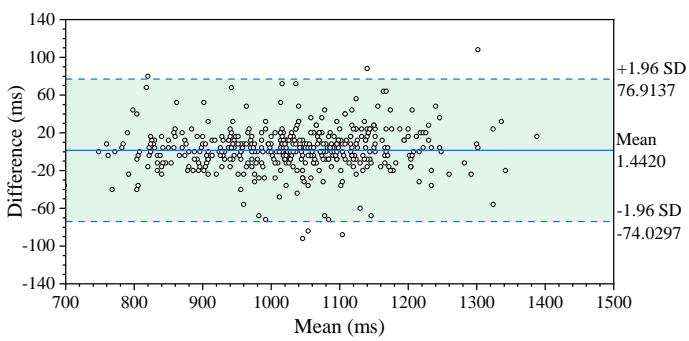

(d) Bland-Altman plot of IBI estimation and ground truth.

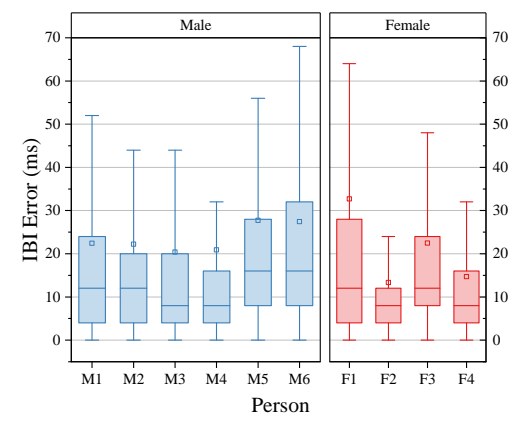

(f) Box plot of IBI error

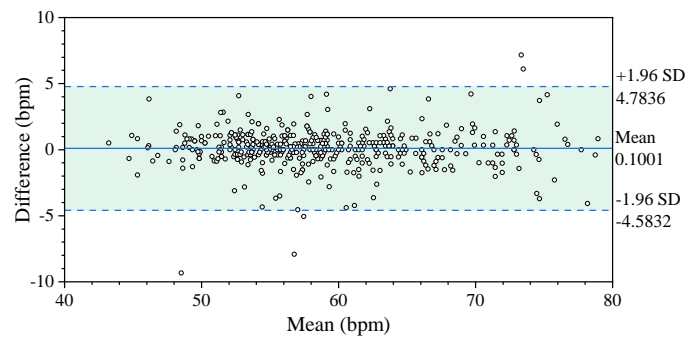

(e) Bland-Altman plot of heart rate estimation and ground truth.

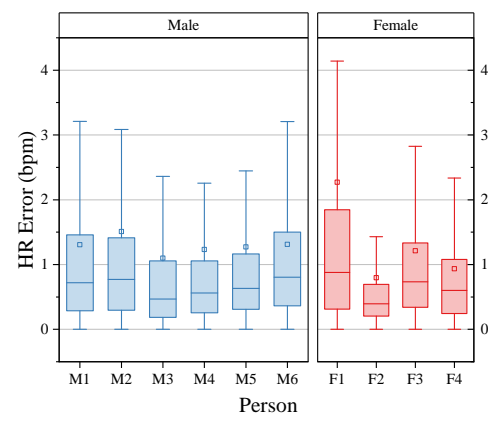

(g) Box plot of HR error

Fig. 8. Overall performance evaluation of RF-Heartbeat. (a) Comparison of our algorithm with ALG1 and ALG2 in IBIe error. (b) Comparison with ALG1 and ALG2 in heart rate error. (c) Comparison with ALG1 and ALG2 in time complexity. (d) Bland-Altman plot of RF-Heartbeat IBI estimation result and ground truth (RR interval of synchronous ECG). (e) Bland-Altman plot of RF-Heartbeat beat-to-beat heart rate estimation result and ground truth (beat-to-beat heart rate derived from synchronous ECG), and the ordinate is difference between them. (f) IBI error box plot of each subject. (g) Heart rate error box plot of each subject.

heartbeat have different sizes, the peak of similarity function $e$ appears at the start of each heartbeat. Thus, also based on dynamic programming, the time complexity of RF-Heartbeat is much lower than ALG1. In addition, ALG1 uses Euclidean distance to measure the similarity between the template and the heartbeat signal, which is easily affected by the amplitude of the heartbeat. However, the amplitude difference among each heartbeat is relatively significant, so it is more robust to use PCCs to quantify the similarity. In addition, when the extracted heartbeat signal series is short, ALG1 is susceptible to interference from incomplete heartbeats at the start and end of the series. RF-Heartbeat is based on the correlation coefficient peak value, which is less disturbed.

The performance of RF-Heartbeat is better than ALG2, while the time complexity is slightly higher than the latter. For the strategy of searching for peaks sequentially, the detection of the current peak depends on the previously detected peaks. When there is a large variation of heartbeat shape, the error in detection of current peak affects the accuracy of subsequent peak detection, as described in section VII. Using dynamic programming can solve this problem and improve the robustness of proposed IBI estimation algorithm.

For each participant, the CDF of HR error and IBI error are shown in Fig. 8(f) and Fig. 8(g), respectively. Results show that for different individuals and different genders, RF-Heartbeat achieves high coverage, low heart rate estimation errors, and low IBI errors.

\section{Robustness to Different Posture}

In order to evaluate the performance of RF-Heartbeat under different lying postures, according to the timestamp, we matched the posture series recorded by SOMNOtouch PSG (as shown in Fig. 7) with synchronous heartbeat signal series. The lying postures of subjects were divided into four categories, lying on the back, lying on the stomach, lying on the left, and lying on the right. Fig. 9(a) and Fig. 9(b) respectively 


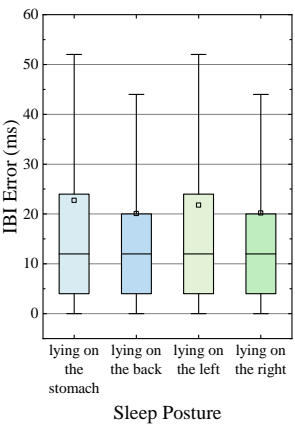

(a) IBI error box plot

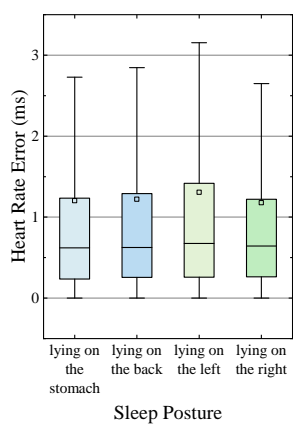

(b) HR error box plot
Fig. 9. Robustness evaluation of RF-Heartbeat in different Lying Postures.

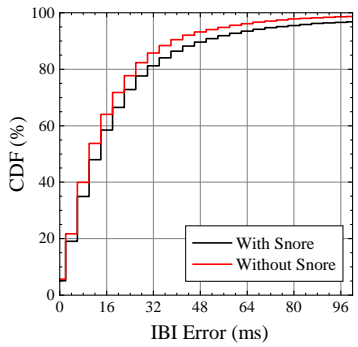

(a) CDF of IBI error

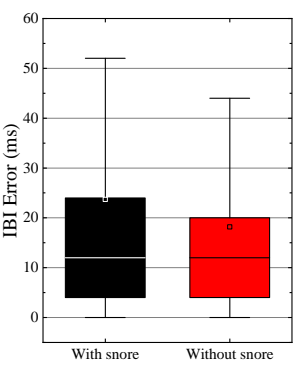

(c) box plot of IBI error

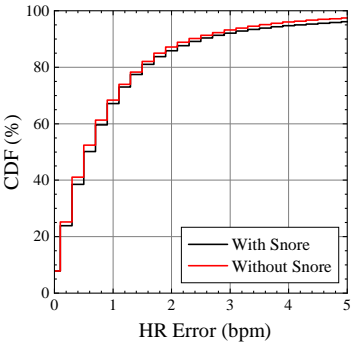

(b) $\mathrm{CDF}$ of HR error

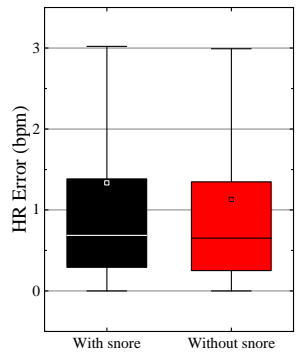

(d) box plot of HR error
Fig. 10. Evaluation of algorithm robustness to snore. These graphs show the comparison of proposed RF-Heartbeat when subjects were snoring or not.

show the CDF of HR error and IBI error when the subjects are in different lying postures. When the subjects were in the four postures, the median IBI errors were all $12 \mathrm{~ms}$, and the median HR were $0.62 \mathrm{bpm}, 0.63 \mathrm{bpm}, 0.67 \mathrm{bpm}$, and 0.64 bpm, respectively. In four postures, the IBI error and HR of the algorithm are all at a low level.

Although when facing the radar, radial displacement caused by heartbeat is the largest. Nevertheless, even when lying on the stomach, this displacement can still be detected from the specific surface of the participants' backs. Millimeterwave radar can detect tiny displacement. In addition, the proposed optimal range bin selection strategy ensures that high SNR heartbeat signals can be extracted in different postures. Therefore, RF-Heartbeat is robust to changes in posture.

\section{Robustness to Snoring During Sleep}

Snoring refers to the vibration of the uvula (palatine) caused by the narrowing of the upper airway during sleep. It is a common phenomenon when the user is sleeping. Snoring can

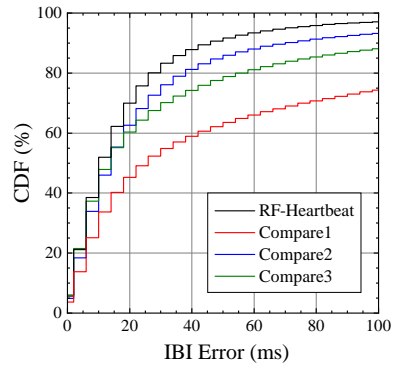

(a) CDF of IBI error

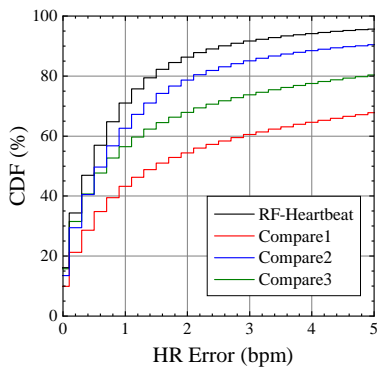

(b) $\mathrm{CDF}$ of HR error
Fig. 11. The effect of proposed range bin selection algorithm, template generation algorithm, and heartbeat segmentation algorithm on RF-Heartbeat performance. Compare1: Result of RF-Heartbeat, when removing range bin selection algorithm. Compare2: Result of RF-Heartbeat, when replacing our template generation algorithm with the method based on Euclidean distance. Compare3: Result of RF-Heartbeat, when replacing our heartbeat segmentation algorithm with the algorithm that sequentially detects peaks.

cause tiny vibrations in the chest and abdomen. Therefore, robustness to snoring needs to be evaluated. SOMNOtouch PSG can analyze pressure airflow collected by nasal cannula to determine whether snoring occurs in a period, as shown in Fig 7.

We compared the HRV estimation result of heartbeat signal series with snore and series without snore, as shown in Fig. 10. Compared to series without snore, the performance of RFHeartbeat when subject snore is slightly worse. However, the accuracy achieved by RF-Heartbeat when a snore occurred can still high, meeting the needs of most applications. In RF-Heartbeat, the bandpass filter eliminates the interference of snore. In addition, a dynamic programming algorithm is selected to calculate the global optimal heartbeat segmentation when the signal has local distortion caused by snore. Results show that RF-Heartbeat has strong robustness to snore.

\section{DISSCUSION}

In this section, we analyze the contributions of RF-Heartbeat and its superiorities over other work.

\section{A. Range Bin Selection Algorithm}

In previous work, the selection of optimal distance units is mainly based on reflection energy [23], [25]. However, because our work needs a smaller range bin $(3.75 \mathrm{~cm})$, the human thoracic cavity is often located in multiple different distance units, and this method often selects the range bin where respiration is most distinct, rather than heartbeat. Because the range bin is small, if the range bin is selected based on reflection energy, even respiration could cause the optimal range bin selected during real-time monitoring to change frequently. Different from previous work, for the heartbeat signal segment without body movement, the optimal range bin selected does not change. Fig. 12 compares the heartbeat signals obtained by using the range bin extraction algorithm in RF-Heartbeat and previous work. Our method can obtain heartbeat signal with better quality compare with previous work [23]. Fig. 11 shows the effect of the range bin selection algorithm on RF-Heartbeat performance. 
(a)

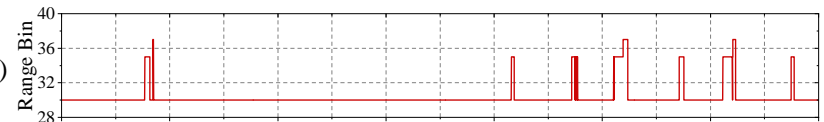

(b)

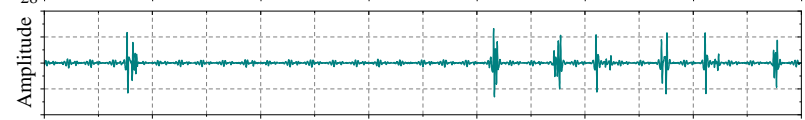

(c)

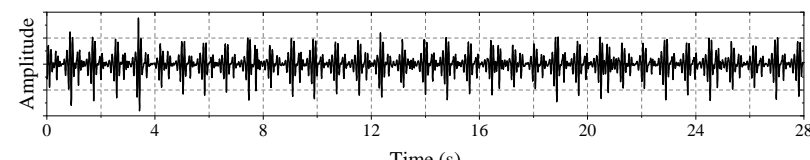

Fig. 12. Comparison of heartbeat signals obtained by RF-Heartbeat and traditional method. (a) Change of range bin with the largest signal amplitude in each chirp. (b) Heartbeat signal obtained by the traditional method that range bin with the largest amplitude of each chirp is selected to extra phase. (c) Heartbeat signal obtained by RF-Heartbeat.

\section{B. IBI Estimation Algorithm}

Template generation: For the heartbeat segment algorithm with template matching, the choice of template determines the accuracy of the algorithm. Some previous work generates the template based on artificial features or supervised learning. These methods are difficult to adapt to the changes of heartbeat shape caused by posture changes, etc. RF-Heartbeat uses clustering, an unsupervised learning algorithm, to generate heartbeat templates. Therefore, for each heartbeat signal series, RF-Heartbeat can generate a template specific to this series. This design makes RF-Heartbeat robust to individual differences, changes in posture, and position. And our IBI estimation algorithm is still applicable to heartbeat signals collected by $\mathrm{CW}$ radar, piezoelectric sensors, etc. Furthermore, according to related research, respiration could lead to changes in amplitude of heartbeat [32]. Thus, unlike other heartbeat template generation algorithms based on clustering, instead of Euclidean distance, we choose PCCs to measure the similarity between heartbeat signal segments. This index is not sensitive to the amplitude of heartbeat, and its magnitude only depends on shape similarity between heartbeat signal segments. Therefore, RF-Heartbeat can obtain a more representative heartbeat template and estimate IBI more accurately. For example, the similarity function $e$ is calculated by using the template generation algorithm in RF-Heartbeat and [11], respectively, and IBI is estimated, as shown in Fig. 13. The effect of our template generation algorithm on RF-Heartbeat performance is shown in Fig. 11. More representative templates are obtained with our method, and there are more distinct peaks in the similarity function $e$.

Heartbeat segmentation algorithm: In some related work, after obtaining a curve resemblant to the similarity function $e$, an algorithm that sequentially detects peaks is often used to estimate IBI. This method assumes that when body movements, each heartbeat has a highly similar shape. However, existing studies have shown that breathing, especially snoring, could cause variation of heartbeat shape [32], [41], [42]. Moreover, variation of IBI does affect the heartbeat shape, as it changes how adjacent heartbeats overlap [32]. As shown in Fig. 14, when there is a certain difference in heartbeat shape obtained by radar, the algorithm based on sequential peak detection

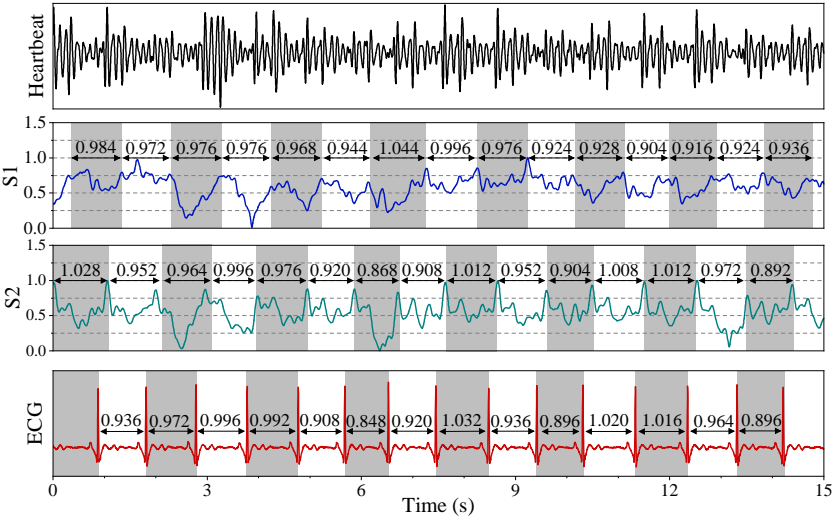

Fig. 13. Example of our template generation algorithm improving RFHeartbeat performance. S1: Similarity function $e$ and IBI estimation result of RF-Heartbeat, when replace our template generation algorithm with method in [11], based on Euclidean distance. S2: Similarity function $e$ and IBI estimation result of RF-Heartbeat.

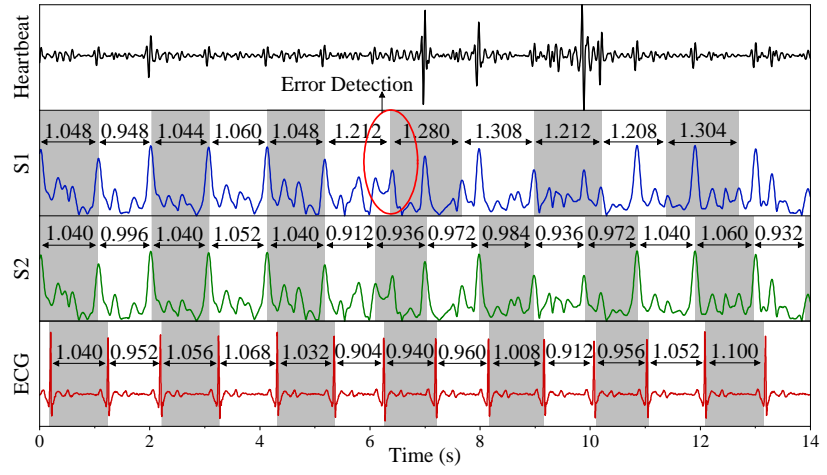

Fig. 14. Example of proposed heartbeat segmentation algorithm improving RF-Heartbeat performance. S1: Similarity function $e$ and IBI estimation result of RF-Heartbeat, when replace our template generation algorithm with algorithm that sequentially detect peaks. S2: Similarity function $e$ and IBI estimation result of RF-Heartbeat.

might detect a wrong peak in a heartbeat with different shapes from other heartbeats, and it results in large IBI estimation error in subsequent signal. Different from these works, we use dynamic programming to enhance the robustness of our template matching algorithm. For each heartbeat signal series, the optimal global segmentation is calculated. This design makes RF-Heartbeat robust to variation of heartbeat shape. Results show that compared with the sequential peak detection algorithm, our method significantly improves the accuracy of IBI estimation, as shown in Fig. 8(a) and Fig. 8(b), and there is not an obvious increase in complexity, as shown in Fig. 8 (c). Fig. 11 shows the effect of proposed Heartbeat segmentation algorithm on RF-Heartbeat performance.

\section{CONCLUSION}

In this paper, we propose RF-Heartbeat, an algorithm using millimeter-wave FMCW radar to accurately and robustly monitor heartbeat in a non-contact approach. As experiment results show, RF-Heartbeat is robust to breathing, snoring during sleep, and other factors that cause signal distortion. Meanwhile, it has the robustness to variation of heartbeat shape and can deal with body movement. Under $91.2 \%$ of time coverage, the median of IBI error is $12 \mathrm{~ms}$, and the median of 
HR error is $0.655 \mathrm{bpm}$. Meanwhile, due to low computational complexity, RF-Heartbeat can monitor heartbeat and estimate IBI accurately in real-time.

RF-Heartbeat has wide applications in clinical space and daily life. Such a system can be implemented in off-theshelf devices, for example, millimeter-wave radars already integrated into millions of smartphones like Pixel 4. RFheartbeat also may be incorporated into a laptop or desktop computer. With the development of IoT devices, it may realize ubiquitous and unobtrusive monitoring.

\section{REFERENCES}

[1] G. A. Mensah, G. A. Roth, and V. Fuster, The global burden of cardiovascular diseases and risk factors: 2020 and beyond, 2019.

[2] L. Fontana et al., Medical research: treat ageing, Nature News, vol. 511, no. 7510 , p. 405, 2014.

[3] M. Zhao, F. Adib, and D. Katabi, Emotion recognition using wireless signals, in Proceedings of the 22nd Annual International Conference on Mobile Computing and Networking, 2016, pp. 95108.

[4] M. Zhao et al., Learning sleep stages from radio signals: A conditional adversarial architecture, in International Conference on Machine Learning. PMLR, 2017, pp. 41004109.

[5] D. Biswas et al., Cornet: Deep learning framework for ppg-based heart rate estimation and biometric identification in ambulant environment, IEEE transactions on biomedical circuits and systems, vol. 13, no. 2 , pp. 282291, 2019

[6] Z. I. Attia et al., An artificial intelligence-enabled ecg algorithm for the identification of patients with atrial fibrillation during sinus rhythm: a retrospective analysis of outcome prediction, The Lancet, vol. 394, no. 10201, pp. 861867, 2019.

[7] D. Hernando et al., Validation of heart rate monitor polar rs 800 for heart rate variability analysis during exercise, The Journal of Strength \& Conditioning Research, vol. 32, no. 3, pp. 716725, 2018.

[8] M. P. Turakhia et al., Rationale and design of a large-scale, app-based study to identify cardiac arrhythmias using a smartwatch: The apple heart study, American heart journal, vol. 207, pp. 6675, 2019.

[9] Y. Jasemian, Elderly comfort and compliance to modern telemedicine system at home, in 2008 Second International Conference on Pervasive Computing Technologies for Healthcare. IEEE, 2008, pp. 6063.

[10] G. Guidoboni et al., Cardiovascular function and ballistocardiogram: a relationship interpreted via mathematical modeling, IEEE Transactions on Biomedical Engineering, vol. 66, no. 10, pp. 29062917, 2019.

[11] Q. Xie et al., A personalized beat-to-beat heart rate detection system from ballistocardiogram for smart home applications, IEEE transactions on biomedical circuits and systems, vol. 13, no. 6, pp. 15931602, 2019.

[12] C. Jiao et al., Non-invasive heart rate estimation from ballistocardiograms using bidirectional $1 \mathrm{stm}$ regression, IEEE Journal of Biomedical and Health Informatics, 2021.

[13] F. Wang et al., Respiration tracking for people counting and recognition, IEEE Internet of Things Journal, vol. 7, no. 6, pp. 52335245, 2020.

[14] C. Shi et al., A 0.065-mm 3 monolithically-integrated ultrasonic wireless sensing mote for real-time physiological temperature monitoring, IEEE transactions on biomedical circuits and systems, vol. 14, no. 3, pp. 412424, 2020

[15] X. Wang, C. Yang, and S. Mao, Phasebeat: Exploiting csi phase data for vital sign monitoring with commodity wifi devices, in 2017 IEEE 37th International Conference on Distributed Computing Systems (ICDCS). IEEE, 2017, pp. 12301239

[16] F. Zhang et al., Your smart speaker can hear your heartbeat! Proceedings of the ACM on Interactive, Mobile, Wearable and Ubiquitous Technologies, vol. 4, no. 4, pp. 124, 2020

[17] C. Ye, K. Toyoda, and T. Ohtsuki, Non-negative matrix factorizationbased blind source separation for non-contact heartbeat detection, in ICC 2019-2019 IEEE International Conference on Communications (ICC). IEEE, 2019, pp. 16.

[18] F. Lin et al., Cardiac scan: A non-contact and continuous heartbased user authentication system, in Proceedings of the 23rd Annual International Conference on Mobile Computing and Networking, 2017, pp. 315328.

[19] C. Will et al., Local pulse wave detection using continuous wave radar systems, IEEE Journal of Electromagnetics, RF and Microwaves in Medicine and Biology, vol. 1, no. 2, pp. 8189, 2017.
[20] A. Ahmad et al., Vital signs monitoring of multiple people using a fmcw millimeter-wave sensor, in 2018 IEEE Radar Conference (RadarConf18). IEEE, 2018, pp. 14501455.

[21] E. Mogi and T. Ohtsuki, Heartbeat detection with doppler radar based on spectrogram, in 2017 IEEE International Conference on Communications (ICC). IEEE, 2017, pp. 16

[22] S. A. T. Hosseini and H. Amindavar, Uwb radar signal processing in measurement of heartbeat features, in 2017 IEEE International Conference on Acoustics, Speech and Signal Processing (ICASSP). IEEE, 2017, pp. 10041007.

[23] F. Adib et al., Smart homes that monitor breathing and heart rate, in Proceedings of the 33rd annual ACM conference on human factors in computing systems, 2015 , pp. 837846 .

[24] M. Mercuri et al., Vital-sign monitoring and spatial tracking of multiple people using a contactless radar-based sensor, Nature Electronics, vol. 2 , no. 6 , pp. 252262, 2019.

[25] F. Wang et al., mmHRV: Contactless heart rate variability monitoring using millimeter-wave radio, IEEE Internet of Things Journal, 2021.

[26] C. Bruser et al., Adaptive beat-to-beat heart rate estimation in ballistocardiograms, IEEE Transactions on Information Technology in Biomedicine, vol. 15, no. 5, pp. 778786, 2011.

[27] L. Sun et al., Remote measurement of human vital signs based on jointrange adaptive eemd, IEEE Access, vol. 8, pp. 68 51468 524, 2020.

[28] T. Sakamoto et al., Feature-based correlation and topological similarity for interbeat interval estimation using ultrawideband radar, IEEE Transactions on Biomedical Engineering, vol. 63, no. 4, pp. 747757, 2015.

[29] Y. Lee et al., A novel non-contact heart rate monitor using impulseradio ultra-wideband (ir-uwb) radar technology, Scientific reports, vol. 8, no. 1, pp. 110, 2018

[30] J. Benesty et al., Pearson correlation coefficient, in Noise reduction in speech processing. Springer, 2009, pp. 14.

[31] B. J. Frey and D. Dueck, Clustering by passing messages between data points, science, vol. 315, no. 5814, pp. 972976, 2007.

[32] J. Paalasmaa, A respiratory latent variable model for mechanically measured heartbeats, Physiological measurement, vol. 31, no. 10, p. 1331, 2010.

[33] D. Amiri et al., Context-aware sensing via dynamic programming for edge-assisted wearable systems, ACM Transactions on Computing for Healthcare, vol. 1, no. 2, pp. 125, 2020.

[34] F. Adib, Wireless systems that extend our senses, Ph.D. dissertation, Massachusetts Institute of Technology, 2016.

[35] M. Kagawa et al., Sleep stage classification by body movement index and respiratory interval indices using multiple radar sensors, in 2015 37th Annual International Conference of the IEEE Engineering in Medicine and Biology Society (EMBC). IEEE, 2015, pp. 76067609.

[36] N. C. Schwertman, M. A. Owens, and R. Adnan, A simple more general boxplot method for identifying outliers, Computational statistics \& data analysis, vol. 47, no. 1, pp. 165174, 2004.

[37] Y. Liu et al., Epidermal mechano-acoustic sensing electronics for cardiovascular diagnostics and human-machine interfaces, Science Advances, vol. 2, no. 11, p. e1601185, 2016.

[38] I. KAKOUCHE et al., New measurement method for respiration and heartbeat estimation using ir-uwb radar, in 2019 6th International Conference on Image and Signal Processing and their Applications (ISPA). IEEE, 2019, pp. 16

[39] S. Tomii and T. Ohtsuki, Heartbeat detection by using doppler radar with wavelet transform based on scale factor learning, in 2015 IEEE International Conference on Communications (ICC). IEEE, 2015, pp. 483488.

[40] P. Hamilton, Open source ecg analysis, in Computers in cardiology. IEEE, 2002, pp. 101104

[41] X. Zhu et al., Real-time monitoring of respiration rhythm and pulse rate during sleep, IEEE transactions on biomedical engineering, vol. 53, no. 12, pp. 25532563, 2006.

[42] F. Yasuma and J.-i. Hayano, Respiratory sinus arrhythmia: why does the heartbeat synchronize with respiratory rhythm? Chest, vol. 125 , no. 2 , pp. 683690, 2004 